IRSTI 47.05 .03

https://doi.org/10.26577/phst.2020.v7.i1.05

\title{
Sea state characterization using fractal techniques on experimental one - dimensional radar signatures
}

\author{
A. Kotopoulis, B. Massinas, G. Pouraimis and P. Frangos* \\ School of Electrical and Computing Engineering, National Technical University of Athens, \\ 9, Iroon Polytechniou Str., 15773 Zografou, Athens, Greece \\ *e-mail: pfrangos@central.ntua.gr
}

\begin{abstract}
This paper presents a novel method of sea state characterization using the 'Mean Fractal Length (MFL)' criterion which is applied to experimental Synthetic Aperture Radar (SAR) one - dimensional signatures (range profiles), provided to our research group by SET 215 Working Group on 'SAR radar techniques'. The MFL criterion uses the 'blanket' technique to provide sea state characterization from SAR radar range profiles. It is based on the calculation of the area of a 'blanket', corresponding to the range profile under examination, and then on the calculation of the corresponding 'Fractal Length' of the range profile. The main idea concerning this proposed technique is the fact that SAR radar range profiles corresponding to different sea states yield different values of 'Fractal Length, FL', namely 'turbulent sea' yields range profiles with larger FL, because of the more 'anomalous behaviour' of the range profiles in that case. As a result, a sea state characterization technique for two different sea states (turbulent and calm sea) is presented in this paper.
\end{abstract}

Key words: sea state characterization, fractal length, synthetic aperture radar.

PACS numbers: 05.45.Df; 84.40.Xb.

\section{Introduction}

Fractals can describe an unlimited number of complex patterns that resemble in different scales and are used as a mathematical tool for a variety of applications, such as image analysis and sorting, applied electromagnetism, etc. [1-6], [924]. The indistinguishable structure on different scales is a basic feature of fractals. Accordingly, fractals can illustrate a certain very strong form of geometric complexity across multiple data sets as well as SAR images. Synthetic Aperture Radar (SAR) images can be considered as fractals for a certain range of magnification [1], [8-10]. In addition, fractal objects have unique properties and features that may be related to their geometric structure [2].

The main objective of this paper is to examine the sea state characterization problem using the 'Mean Fractal Length' (MFL). The MFL criterion is a 'product' of the 'Modified Fractal Signature' (MFS) method, which has been applied in the past to real Synthetic Aperture Radar (SAR) images, using the 'blanket' technique, in order to provide useful information about SAR image classification, as reported by Malamou et. al. [1], Peleg et. al. [3] and Tang et. al. [4].

This paper uses the recorded sea clutter radar data which were collected during the 'NEMO 2014' trials in Taranto, Italy, using FFI (i.e. 'Norwegian Institute of Defense', Oslo, Norway) PicoSAR Xband radar as input to a specific SET Working Group. The experiment took place in the Taranto bay in southern Italy on 23 and 24 September 2014. The first day the weather was quite windy, thus creating a rather turbulent sea, in comparison with the second day, during which the sea surface was almost calm.

2 Problem geometry, experimental 1D radar data sets, the 'Strip' fractal technique and preliminary numerical results using the 'Strip' technique

The geometry of the sea state characterization problem is shown in Figure 1. Here, a helicopter 
(with PicoSAR radar inside) rises vertically, while maintaining its steady position (latitude and longitude), and transmits electromagnetic (EM) radar pulses towards the sea. In addition, it records the azimuth angle with high sampling density in grazing angle.

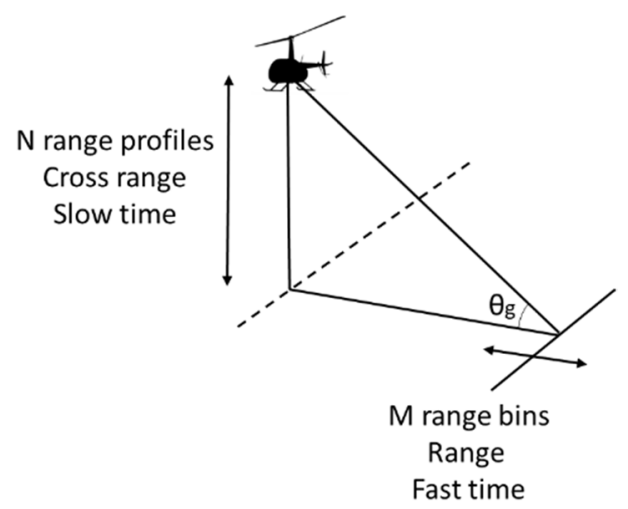

Figure 1 - Geometry of sea state characterization problem, where the helicopter rises vertically transmitting PicoSAR radar electromagnetic (EM) pulses towards the sea

During the experiment, performed by FFI in September 2014 (NEMO trials), the helicopter kept low vertical velocity and negligible horizontal velocity (helicopter movement from down to up). The first day (23/9/2014), the wind speed was reported in the range of 10 to $12 \mathrm{~m} / \mathrm{s}$ (rather high wind speed) and the helicopter pilot kept the direction of the antenna beam up-wind (i.e. direction of radar pulses - EM wave propagation in the opposite direction of the wind speed), within a $20^{\circ}$ window in the horizontal (azimuthal) direction, as grazing angles $\theta \mathrm{g}$ (see Figure 1) scanned from $3^{\circ}$ to $55^{\circ}$. The time of the full grazing angle span was around 5 minutes.

During the second day (24/9/2014), the wind speed was very low $(1-2 \mathrm{~m} / \mathrm{s}$, which sometimes died out locally) and the range of grazing angles was from $4^{\circ}$ to $54^{\circ}$ with a slight drift in azimuth pointing angle of the bore sight of no more than $20^{\circ}$.
Figures $2 \mathrm{a}$ and $2 \mathrm{~b}$ show representative radar range profiles (1D radar signatures) from 'Day 1' (23-9-2014, 'turbulent sea') at grazing angles of $\theta_{\mathrm{g}}=$ 350 (Figure on 2a), and from 'Day 2' (24-9-2014, 'calm sea'), for $\theta_{\mathrm{g}}=350$ (Figure $2 \mathrm{~b}$ ).

As follows from Figure 2a, on 23/9/2014 the grazing angle was chosen, from $35^{\circ}$ to $36^{\circ}$ (for 'turbulent sea,,), with corresponding maximum value of approximately 15,000.

Additionally, as it can be seen from Figure $2 b$, during the following day of 24/9/2014, for the same grazing angles of $35^{\circ}$ to $36^{\circ}$, but for ,calm sea in this case, the approximate maximum value of the range profiles was approximately equal to 1,600.

The 'Mean Fractal Length (MFL)' criterion was used for the sea state determination, which computes the mean of the 'Fractal Length' of the range profile, for turbulent and calm sea, and at grazing angles of $35^{\circ}$ and $40^{\circ}$ as well. The MFL is given by eq. (1):

$$
<F L>=\frac{1}{M} \sum_{m=1}^{M}(F L)_{m}
$$

In this Section, it remains to explain how the 'Fractal Length, FL' is calculated. For this reason, the 'blanket technique' will be described briefly [1], [3].

First, for measuring the lengths of irregular curves, S. Peleg et. al. used a 'Mandelbrot method' [3]. In this example, the curve is shown at Figure 3 (inner curve, out of 3 curves). Considering all points with distances to this curve no more than $\varepsilon$, a strip of width $2 \varepsilon$ is formed. This strip creates a 'strip' (2D case examined here, or 'blanket', in the corresponding 3D case), above and below the inner curve, as shown at Figure 3, which means that all points at distance $\varepsilon$ cover the curve within a 'strip' of thickness $2 \varepsilon$. According to S. Peleg et. al. [3], the 'upper' and 'lower' curves of the 'strip' are provided by the following equations:

$$
\begin{gathered}
u_{\varepsilon}(i, j)=\max \left\{u_{\varepsilon-1}(i, j)+1, \max _{|(m, n)-(i, j)| \leq 1} u_{\varepsilon-1}(m, n)\right\} \\
b_{\varepsilon}(i, j)=\max \left\{b_{\varepsilon-1}(i, j)-1, \max _{|(m, n)-(i, j)| \leq 1} b_{\varepsilon-1}(m, n)\right\}
\end{gathered}
$$

Eq. (2) ensures that the new upper curve $u_{\varepsilon}$ is higher at least by one than $u_{\varepsilon-1}$, and also at a distance of at least one of $u_{\varepsilon-1}$ in the horizontal and vertical directions [3]. 

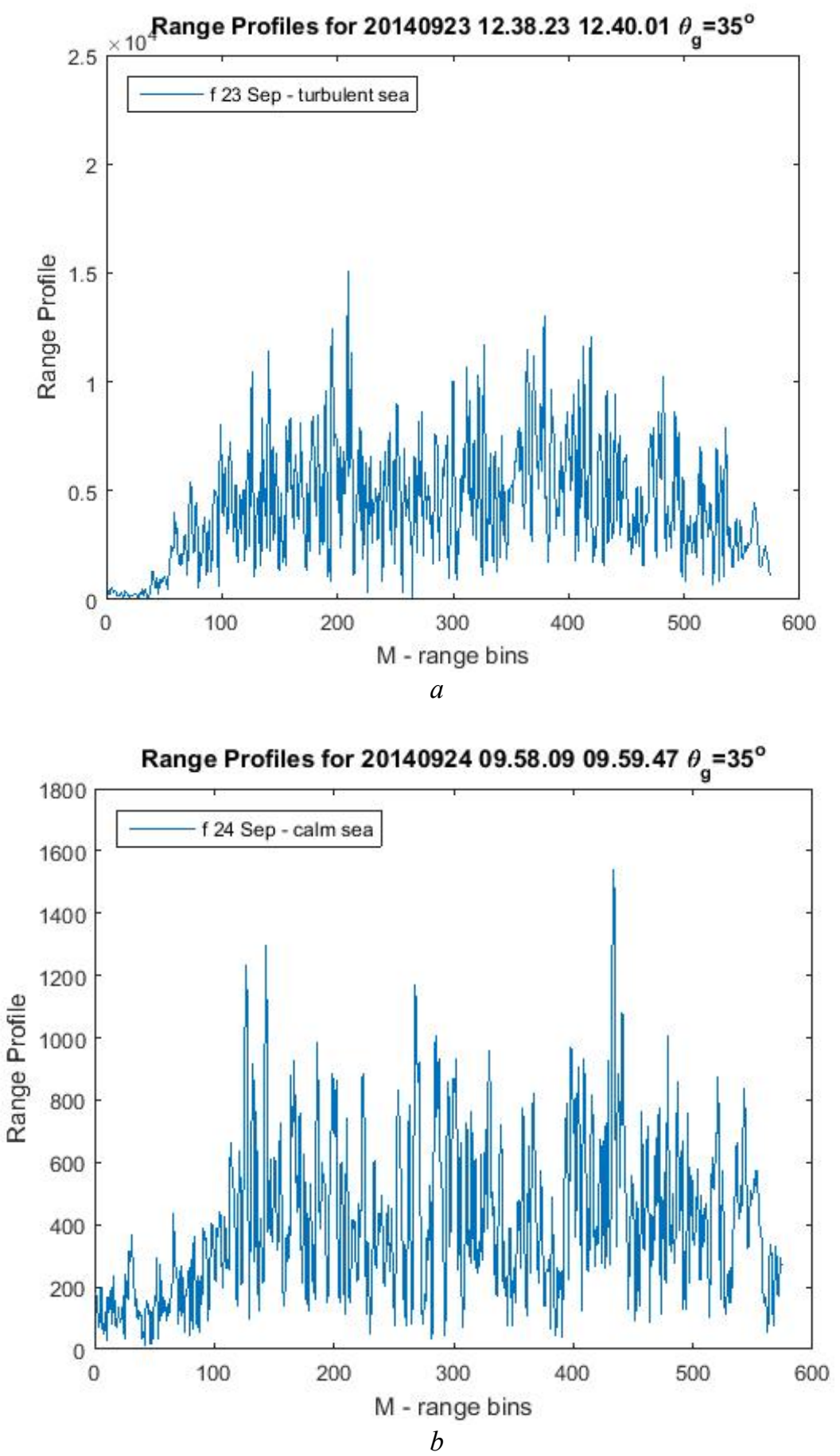

Figure 2 - Representative PicoSAR radar range profiles:

(a) Day 1(turbulent sea) grazing angle $\theta \mathrm{g}=35^{\circ}$, (b) Similarly, but for Day 2 (calm sea) 


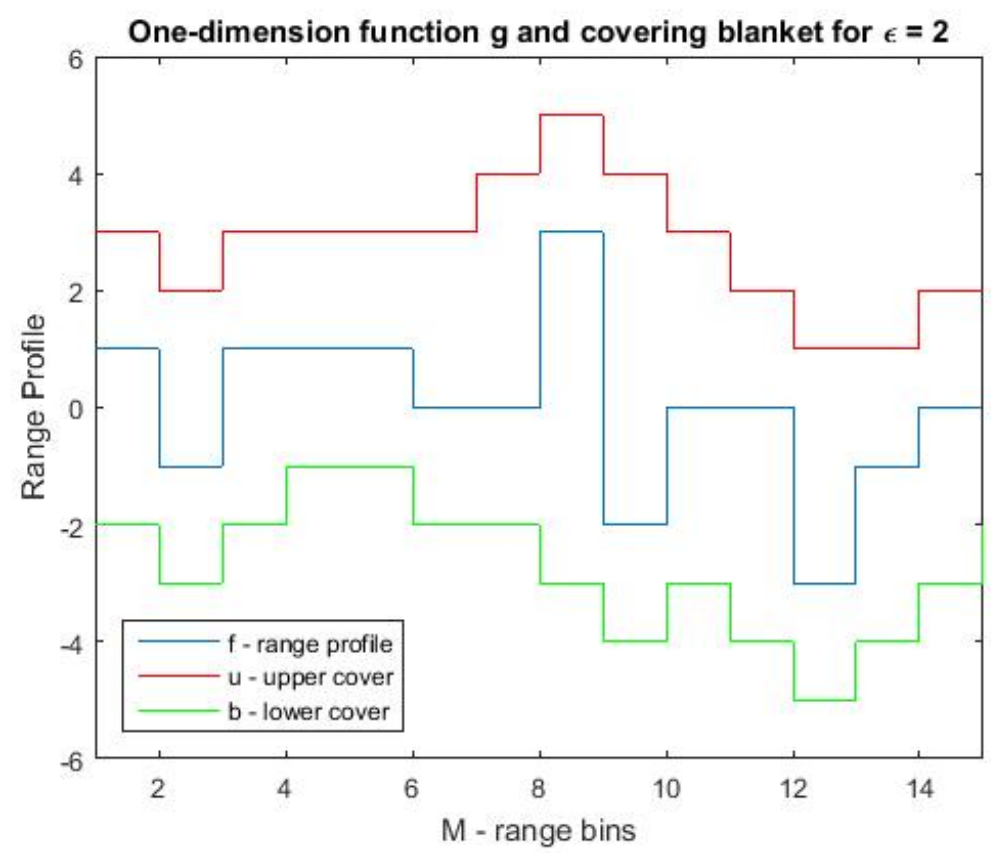

Figure 3 - One-dimensional (1D) function $g$ and the 'upper' and 'lower' curves of the strip for iteration number $\varepsilon=2$

The 'area' $v_{\varepsilon}$ of the 'strip' is calculated from $u_{\varepsilon}$ and $b_{\varepsilon}$ by :

$$
v_{\varepsilon}=\sum_{i . j}\left(u_{\varepsilon}(i, j)-b_{\varepsilon}(i, j)\right)
$$

The 'fractal length, FL' $\mathrm{L}(\varepsilon)$ of the curve is approximately calculated through the subtraction of the strip areas of radii $\varepsilon$ and $\varepsilon-1$ divided by 2 , or from the area of the 'strip' divided by $2 \varepsilon$, as shown below :

$$
\begin{gathered}
L_{\varepsilon}=\frac{\left(A_{\varepsilon}-A_{\varepsilon-1}\right)}{2} \\
L_{\varepsilon}=\frac{A_{\varepsilon}}{2 \varepsilon}
\end{gathered}
$$

The fractal length $\mathrm{L}(\varepsilon)$ as a function of the 'resolution' $\varepsilon$ ( $\varepsilon=1$ corresponds to 'full resolution), for the curve of Figure 3 [3], is shown at Figure 4, on a log-log scale (here the plot consists of straight segments, because the curve is ideally fractal. In contrast, the curve would not have to be straight for non - fractal curves [3]).

In addition, previous research by Malamou et. al. [1], regarding use of the 'Modified Fractal Signature (MFS)' method, which was applied to real Synthetic Aperture Radar (SAR) images, used the 'blanket' technique (in 3D case), to provide useful information for SAR image classification.

\section{Sea State Characterization Results using the 'Mean Fractal Length (MFL)' criterion}

The 'Mean Fractal Length (MFL)' criterion is used for characterization of the sea state. The 'Mean Fractal Length (MFL)' Criterion computes the mean of the Fractal Length of the range profile, according to eq. (1). Then, numerical calculations similar to the above were performed, and the results are presented at Figures $5 \mathrm{a}$ and $5 \mathrm{~b}$. 


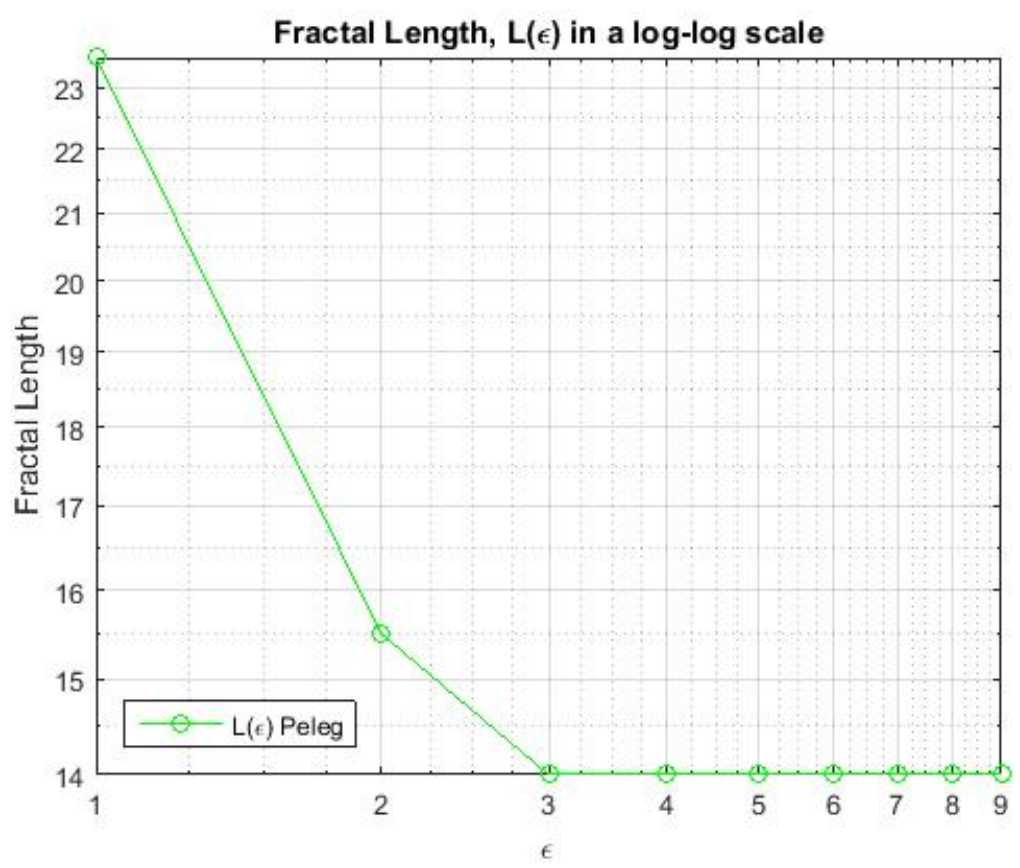

Figure 4 - Fractal length $L(\varepsilon)$ as a function of resolution $\varepsilon(\varepsilon=1$ corresponds to 'full resolution') in log-log scale for one - dimensional (1D) curve g.
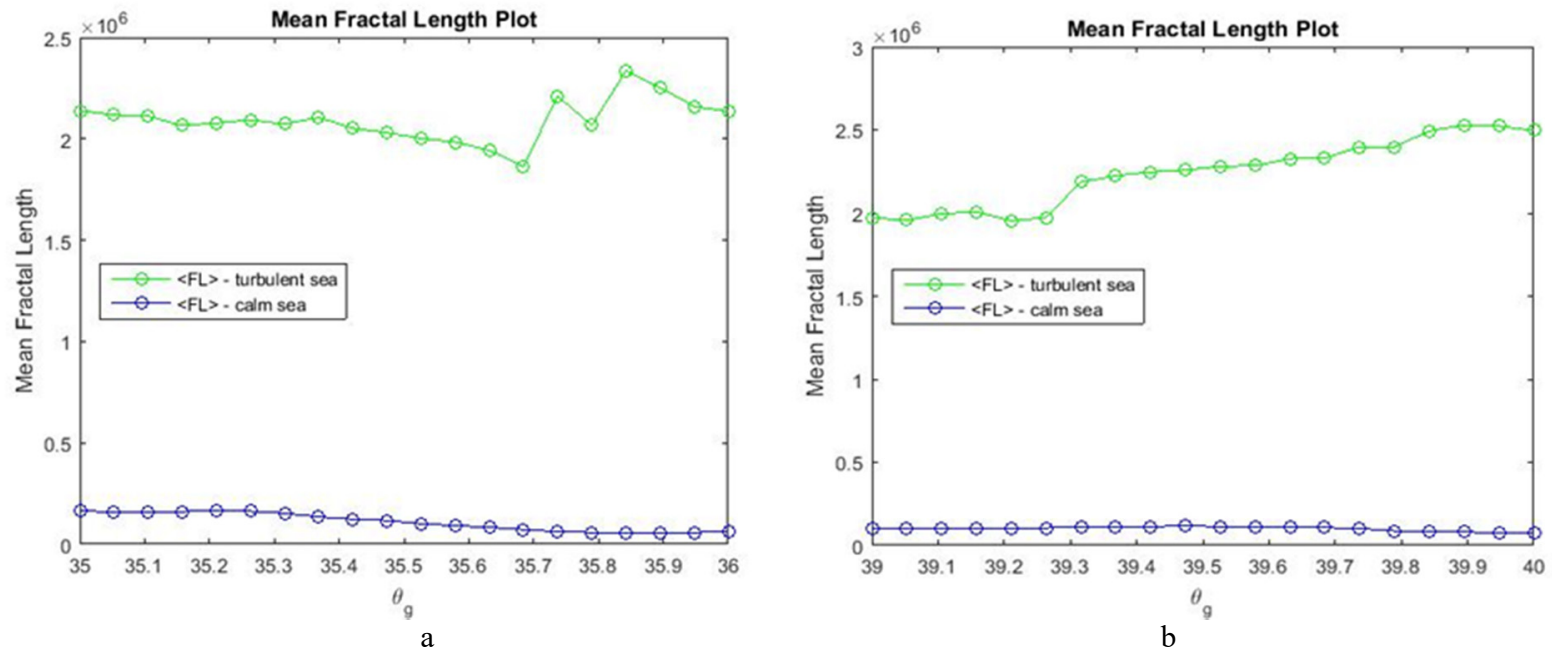

Figure 5 - 'Mean Fractal length' (MFL) values of radar range profiles at different sea state [turbulent (green lines) and calm sea (blue lines)], for grazing angles $35^{\circ}$ to $36^{\circ}$ (a) and $39^{\circ}$ to $40^{\circ}$ (b)

The results of Figure 5 show that the MFL values of radar range profiles during the turbulent sea state are significantly larger than the corresponding values at calm sea, as shown at Table 1 .

Finally, we introduce here a useful index for 'sea state determination', which we call 'sea state index' (SSI), as the ratio of the previously calculated 'MFL value' for 'turbulent sea' over the corresponding 'MFL value' for 'calm sea' (that is, the 'MFL value' for 'calm sea' is chosen here as the 'reference value'). Then, the corresponding results for SSI are shown at Table 2, below.

Concluding with the above criterion for sea state characterization by using radar range profiles (1D radar signatures), it is evident, from physical intuition that the 'mean fractal length', (MFL) is a reliable criterion for 'real time' sea state characterization, in practical circumstances (because 
of the presence of additive noise in 'real life' scenarios, etc.).

Table 1 - MFL values results for different sea states

\begin{tabular}{|c|c|c|}
\hline MFL Date & $\begin{array}{c}\text { 23 Sep 2014 } \\
\text { (turbulent sea) }\end{array}$ & $\begin{array}{c}\text { 24 Sep 2014 (calm } \\
\text { sea) }\end{array}$ \\
\hline$\theta \mathrm{g}=35^{\circ}-36^{\circ}$ & $2,090,761$ & $110,631.9$ \\
\hline$\theta \mathrm{g}=39^{\circ}-40^{\circ}$ & $2,241,509$ & $100,038.9$ \\
\hline
\end{tabular}

Table 2 - MFL sea state index (SSI) for different grazing angles

\begin{tabular}{|c|c|}
\hline & SSI \\
\hline$\theta \mathrm{g}=35^{\circ}-36^{\circ}$ & 18.89 \\
\hline$\theta \mathrm{g}=39^{\circ}-40^{\circ}$ & 22.40 \\
\hline
\end{tabular}

\section{Conclusions}

To summarize, for the characterization of the sea state from experimental 1D radar signatures (range profiles), the 'mean fractal length' (MFL) criterion was used. The corresponding recorded sea clutter radar data were collected during the 'NEMO 2014' trials in Taranto, Italy, 23-24/9/2014. An Xband PicoSAR airborne radar was used for that purpose by FFI (i.e. 'Norwegian Institute of Defense', Oslo, Norway)
The above criterion was found to be suitable and it can be used for sea state characterization. Other criteria for sea state determination, which are, however, of less importance than that described above, will also be presented during our presentation at the Conference.

\section{Future Research}

In our future related research, we intend to concentrate on more accurate sea state characterization using a variety of sea surface radar range profiles, i.e. in a variety of sea state conditions.

Finally, sea state characterization using fractal characteristics of SAR radar images (i.e. 2D SAR radar signatures) may be used, instead of $1 \mathrm{D}$ radar signatures, examined here.

\section{Acknowledgments}

The authors (AK, BM, GP and PF) would like to thank SET-215 Working Group, and FFI Institute (i.e. 'Norwegian Institute of Defense', Oslo, Norway), in particular, for providing to us the real recorded sea clutter radar data which were collected during the 'NEMO 2014' trials in Taranto, Italy, and are shown in Figures 2 above.

\section{References}

1. Malamou A., Pandis C., Frangos P., Stefaneas P., Karakasiliotis A., Kodokostas D. Application of the modified fractal signature method for terrain classification from synthetic aperture radar images // Electronics and Electrical Engineering Journal - 2014 - V.20 - No.6 - P.118-121.

2. Falconer K. J. Fractal geometry: Mathematical foundations and applications. J. Wiley and Sons. 1990.

3. Peleg S., Naor J., Hartley R., Avnir D. Multiple resolution texture analysis and classification // IEEE Transactions on Pattern Analysis and Machine Intelligence. - 1984. - V.PAMI-6. -No.4.

4. Tang Y., Ma H., Xi D., Mao X., Suen C. Modified fractal signature (MFS): A new approach to document analysis for automatic knowledge acquisition // IEEE Transactions on Knowledge and Data Engineering. -1997. - V.9. No.5. -P. 747-762.

5. Mandelbrot B. The fractal geometry of nature. New York: W. H. Freeman and Company. 1977.

6. Jaggard D. L., Jaggard A.D., Frangos P. Fractal electrodynamics: surfaces and superlattices, in frontiers in electromagnetics. In edited by D. Werner and R. Mittra - IEEE Press - 2000 - pp. 1-47

7. Beckmann P., Spizzichino A. The scattering of electromagnetic waves from rough surfaces. Norwood, MA: Artech House. 1987.

8. Malamou A., Karakasiliotis A., Kallitsis E., Boultadakis G., Frangos P. Application of a fully automatic autofocusing algorithm for post - processing of synthetic aperture radar images based on image entropy minimization // Electronics and Electrical Engineering Journal. - 2013. - V.19 - No.6 - P.95-98

9. Pouraimis G., Kotopoulis A., Malamou A., Kallitsis E, Frangos P. Characterization of three dimensional rough fractal surfaces from backscattered radar data // Elektronika ir Elektrotechnika. -2017. -V. 23 - No. 4. - P. $1-5$.

10. Kotopoulis A., Malamou A., Pouraimis G., Kallitsis E., Frangos P. Characterization of rough fractal surfaces from backscattered radar data. // Elektronika ir Elektrotechnika. - 2016. -V. 22 -P. 1-6. 
11. Ampilova N., Soloviev I. On digital image segmentation based on fractal and multifractal methods // CEMA ${ }^{\text {ee }} 15$ Conference Proceedings. - 2015. -P. 14-17.

12. Iodice A., Natale A., Riccio D. Kirchhoff scattering from fractal and classical rough surfaces: Physical interpretation // IEEE Transactions on Antennas and Propagation. - 2013. - V. 61 -No. 4. -P. 1-7.

13. Li B., Xu Y., Zhang J., Cui L. Study on calculation models of curve fractal dimension // Sixth International Conference on Natural Computation (ICNC 2010). -2010. -P. 22-25.

14. Martorella M., Berizzi F., Dalle Mese E. On the fractal dimension of sea surface backscattered signal at low grazing angle // IEEE Transactions on Antennas and Propagation. - 2004. -V.52. - No. 5. P.1-8.

15. Berizzi F., Dalle Mese E., Pinelli G. One-dimensional fractal model of the sea surface // IEE Proc.-Radar, Sonar, Navig. - 1999 - V. 146 - No.1.-P.1-5.

16. Savaidis S., Frangos P., Jaggard D., Hizanidis K. Scattering from fractally corrugated surfaces: An exact approach // Optics Letters. - 1995. - V. 20. - No. 23. - P. 2357.

17. Berizzi F., Dalle-Mese E. Scattering from a 2-D sea fractal surface: Fractal analysis of the scattered signal //IEEE Transactions on Antennas and Propagation. -2002. -V. 50. -No. 7. - P. 1-8.

18. Chen J., Lo T., Leung H., Litva J. The use of fractals for modeling EM waves scattering from rough sea surface // IEEE Transactions on Geoscience and Remote Sensing. - 1996. -V. 34. - No. 4. -P. 1-5.

19. Lin N., Lee H.P., Lim S.P., Lee K.S. Wave scattering from fractal surfaces // Journal of Modern Optics. - 2007. -V.3. -P.344.

20. Lo T., Leung H., Litva J., Haykin S. Fractal characterisation of sea-scattered signals and detection of seasurface targets // IEE Proceedings-F. -1993. -V.140. - No. 4.-P. 1-5.

21. Jaggard D., Sun X. Scattering from fractally corrugated surfaces // Journal of the Optical Society of America A. - 1990. - V.7. - P.1131-1139.

22. Zaleski A. Fractals and the weierstrass Mandelbrot function // Rose-Hulman Undergraduate Mathematics Journal. - 2012. - V.13. - No.2. - P.80 - 113.

23. Berizzi F., Dalle-Mese E. Fractal analysis of the signal scattered from the sea surface // IEEE Transactions on Antennas and Propagation. - 1999. - V. 47. - No. 2.

24. Xin R., Li-Xin G. Fractal characteristics investigation on electromagnetic scattering from 2-D Weierstrass fractal dielectric rough surface // Chinese Physics B. - 2008. - Vol.17. - No.8. -P.1-7. 